\title{
Histogenesis and Histomorphometric study of Human Fetal Small Intestine
}

\author{
Salva MN', Chandni Gupta ${ }^{1 *}$, Arvind Kumar Pandey ${ }^{1}$, Nitesh Kumar², Sushma \\ R Kotian ${ }^{1}$, Sneha G Kalthur ${ }^{1}$
}

\author{
OPEN ACCESS \\ Citation: Salva M N, Chandni Gupta, \\ Arvind Kumar Pandey, et al. \\ Histogenesis and Histomorphometric \\ study of Human Fetal Small Intestine. \\ Ethiop J Health Sci. 2019; 29(6):689. \\ doi:http://dx.doi.org/10.4314/ejhs.v29 \\ i6.5 \\ Received: July 17, 2019 \\ Accepted: August 7, 2019 \\ Published: November 1, 2019 \\ Copyright: (C2019 Salva MN, et al. This \\ is an open access article distributed under \\ the terms of the Creative Commons \\ Attribution License, which permits \\ unrestricted use, distribution, and \\ reproduction in any medium, provided the \\ original author and source are credited. \\ Funding: Nil \\ Competing Interests: The authors \\ declare that this manuscript was approved \\ by all authors in its form and that no \\ competing interest exists. \\ Affiliation and Correspondence: \\ ${ }^{1}$ KMC Manipal, Manipal, Manipal \\ Academy of Higher Education, \\ Manipal, India, 576104 \\ ${ }^{2}$ MCOPS, Manipal, Manipal Academy \\ of Higher Education, Manipal, India, \\ 576104 \\ *Email: chandnipalimar@gmail.com
}

\section{ABSTRACT}

Background: Intestine plays a major role for the normal growth of the fetus during the prenatal period. The process of the embryonic development is not quantified histologically. Therefore the main aim of the study was to measure the thickness of all part of the wall of the small intestine that are mucosa, submucosa and muscularis externa and to look for the appearance of the Brunner's glands and Peyer's patches in the submucosa of duodenum and ileum.

Methods: The present study was carried out on 30 fetuses of gestational ages ranging from 11-36 weeks. Ten fetuses from each trimester were used in the study. Fetal small intestine were dissected carefully, and were separated as duodenum, jejunum \& ileum and fixed in formalin solution. The tissue was processed for histology and then slides were stained with Haematoxylin and Eosin. The microscopic features were noted using light microscope. Results: The thickness of the mucosa, submucosa and the muscularis externa was observed to be increased in first trimester, decreased in the second trimester and again increased in the third trimester, which could be because of the increase cell turnover and the arrangement of the collagen fibers as to support the mucosa and the muscularis externa.

Conclusion: Thus, the knowledge of the histogenesis and histomorphometry of the human fetal small intestine is crucial for the adult gastroenterologist to appreciate, because of the potential for these early life events to affect the responsiveness of the intestine to physiological or pathological challenges in later life.

KEYWORDS: Small intestine, Brunner's gland, Peyer's patches, Thickness.

\section{INTRODUCTION}

The small intestine is a long tube like structure, measuring about 5-6 meters in length, which is to be differentiated into three parts from cranio-caudal sequence, the duodenum, which measures about $25 \mathrm{~cm}$ in length, the jejunum, which is about two meters \& the ileum, about three meters in length. (1) It begins to develop early in the fourth week of development and by 12-14 weeks, small intestine assumes its definite shape and reaches its final site in the abdomen. (2) This intestine appears to be highly coiled in the abdomen. The human

DOI: http://dx.doi.org/10.4314/ejhs.v29i6.5 
small intestine is biochemically and morphologically more mature than other mammals. The small intestine is the principal site for absorption of digestion products. Through its enteroendocrine cells the small intestine secretes hormones. Completion of the digestion of food particles takes place in the small intestine. Hence, the intestine plays a crucial role for the normal growth of the fetus during the prenatal period.

The small intestine is a derivative of the midgut \& partly forgut. Its development in the fetal life take up a drastic change i.e., the whole of the midgut from which almost whole part of small intestine is derived undergo rotation twice. In the fifth week of development, the primary loop of intestine is seen to be herniated in the umbilical cord, which without change remains in the cord up to $10^{\text {th }}$ week. After this, the intestinal loop undergoes anti-clockwise rotation which is to right at 90 degree. (2) The intestinal loop rapidly grows longer and coils on its own. Then, this again undergoes 180 degree rotation which is again in anti-clockwise direction, where it now takes up its fixed position in the abdomen. (2)

Histologically, the walls of the small intestine are composed of distinct four layers which are, the mucosa, the submucosa, the muscularis externa and serosa. (1) The mucosa and the submucosal layers of the intestine shows different special features. The mucosal layer of all three parts of intestine exhibits different patterns of arrangement of villi. (1) The submucosal layer of duodenum and ileum has special distinguishing features which are the appearance of Brunner's glands (BG) in the duodenum and the Peyer's patches (PP) in the ileum. Apart from these special features specifically, the rest of the intestinal layer shows almost the similar features. Human fetal small intestine start differentiating much earlier in gestation. In the $1^{\text {st }}$ trimester, 10-11 week human fetal mucosal lining is made up of relatively stratified epithelial cells. (3) The mesenchymal invagination starts appearing at the basal part of the epithelium at this age, which later produces the primordial villi or folds. Villi appear 1week prior in the proximal part of small intestine than in the distal part. (3) By 13th week, the transition from stratified epithelium to simple columnar epithelium takes place. It takes quite a lot of time for the morphogenesis of villi in human fetuses. Absorptive cells that line the villi appear between 14-20 weeks of development.
The differentiation of small intestine begins from its distal end to its proximal end, a design which is a distinctive feature of intestinal ontogenesis. (4)

Mucosal layer development: Before the development of rudimentary villi, stratified epithelium lines the luminal surface of the intestine and it was about four layers thick. At around 7 to 8 weeks villi start forming in the duodenum and proximal jejunum as round outgrowth of epithelium and the lumen of the duodenum gets totally filled with rapidly multiplying epithelium. While same quick multiplication was happening in the lower region but in that area it does not completely blocked the lumen after duodenum. Later by $9^{\text {th }}$ to $10^{\text {th }}$ week the lumen of duodenal gets completely patent. Later when the villi started increasing in length in the proximal segment villi begin to appear in more distal part of small intestine. Later villi are present all over the jejunum, but they are undeveloped in the proximal ileum, and are absent in the distal part of ileum. At 14 weeks, the whole of small intestinal mucosa is lined by villi. At 8 to 10 weeks the epithelial cells show many microvilli. By the 10th to 12 th week the basement lamina is well developed embryonic intestinal crypts start appearing at 10 to 12 weeks of gestation. Muscularis mucosa develops by 20 weeks. (4)

Submucosa: At 14 weeks Brunner's glands start developing in duodenum. The circular folds of kerkring appear at around 14 weeks in the midsmall intestine and later they are present throughout the small intestine by 32 weeks of gestation. Meissner's plexus appears in submucosa at around 13 weeks. Peyer's patches in ileum are well developed by the 20 weeks of gestation. (4)

Muscularis externa: Auerbach's plexus appears at 9 weeks of gestation in the small intestine. Muscle layer in the intestine develops by 12 weeks. (4)

There are many complex processes which combined to produce functionally significant modifications during the late intrauterine life in the intestinal morphology so as to make the infant ready for early feeding on high-fat milk, and then weaning onto lower fat-but higher carbohydratecontaining solid foods. So, if we know the changes which happened in the last trimester of pregnancy they could help us to recognize the early age-dependent approach to nutritional disease state. (3) 
Although, the development of the intestines is well documented in the available literature, the histogenesis in humans is seldom explained. Further the process of the embryonic development is not quantified histologically. The aim of the present study was to evaluate the histomorphometric changes in all parts of small intestine as well as the appearance of BG and PP.

\section{MATERIALS AND METHODS}

The present study was carried out in the Department of Anatomy, Kasturba Medical College, Manipal, Manipal Academy of Higher Education, Manipal, with due permission from Ethical Committee (Registration No. ECR/146/lnst/KA/2013) from April 2014-April 2015. The study was carried out on 30 fetuses of gestational ages ranging from 11-36 weeks. Ten fetuses from each trimester were used in the study. Fetuses with gross anomalies were excluded. Fetal small intestine were dissected carefully, and were separated as duodenum, jejunum \& ileum and fixed in $10 \%$ formalin solution. Further, dehydration was done using increased grades of alcohol, i,.e tissues were kept in $50 \%$ alcohol for 24 hours followed by $70 \%$ for 24 hours, then $90 \%$ alcohol for 8 hours and finally in absolute alcohol for 6-8 hours. The tissues are then immersed in xylol for clearing until its transparency is attained. The tissues are kept for impregnation in molten paraffin wax tanks for 2 hours. The paraffin embedding was made upon the procedures of Carleton. (5) Serial sections were taken and standardized method of mounting and fixing were used. The tissue slides were stained with Haematoxylin and Eosin (H\&E). The microscopic features were observed using light microscope. The image analyzer software Image Pro Premier 9.1 was used to measure the histomorphometry of the small intestine as well as to capture the images. The mean, standard deviation minimum and maximum of thickness was calculated out of 10 specimens from each trimester. Values are analyzed using two way ANOVA followed by Tukey's post hoc test, using Graph Pad Prism version 5 , evaluation version.

\section{RESULTS}

The thickness of mucosa, submucosa and muscularis externa is described following the trimesters of the fetus development and is shown in Table 1.

Table 1: Showing the thickness of the mucosa, submucosa and muscularis externa in the microscopic images of the parts of the small intestine.

\begin{tabular}{|c|c|c|c|c|c|c|c|c|c|}
\hline \multirow[t]{3}{*}{ Particulars } & \multicolumn{9}{|c|}{ MUCOSA ( thickness in mm ) } \\
\hline & \multicolumn{3}{|c|}{ DUODENUM } & \multicolumn{3}{|c|}{ JEJUNUM } & \multicolumn{3}{|c|}{ ILEUM } \\
\hline & 1st tri & 2nd tri & 3rd tri & 1st tri & 2nd tri & 3rd tri & 1st tri & 2nd tri & 3rd tri \\
\hline MEAN & 738.8 & 591.0 & 947.2 & 663.3 & 604.8 & 1102.0 & 707.3 & 684.7 & 705.8 \\
\hline MINIMUM & 115.0 & 393.8 & 734.9 & 317.4 & 338.4 & 804.9 & 360.9 & 425.9 & 553.8 \\
\hline MAXIMUM & 1579.9 & 950.5 & 1210.1 & 1335.9 & 1114.9 & 1306.4 & 1169.0 & 932.3 & 990.9 \\
\hline S D & \multirow{2}{*}{\multicolumn{9}{|c|}{ SUBMUCOSA ( thickness in mm) }} \\
\hline \multirow[t]{3}{*}{ Particulars } & & & & & & & & & \\
\hline & \multicolumn{3}{|c|}{ DUODENUM } & \multicolumn{3}{|c|}{ JEJUNUM } & ILEUM & & \\
\hline & 1st tri & 2nd tri & 3 rd tri & 1st tri & 2nd tri & 3rd tri & 1 st tri & 2nd tri & 3rd tri \\
\hline MEAN & 410.2 & 538.8 & 662.3 & 395.2 & 337.0 & 590.0 & 358.5 & 301.1 & 366.1 \\
\hline MINIMUM & 340.4 & 261.8 & 474.9 & 221.1 & 201.0 & 282.5 & 150.1 & 191.6 & 241.4 \\
\hline MAXIMUM & 730.7 & 715.6 & 1059.2 & 617.2 & 576.5 & 957.2 & 558.3 & 476.3 & 675.5 \\
\hline S D & 124.9 & 141.3 & 212.0 & 151.7 & 114.8 & 229.1 & 133.3 & 91.6 & 131.2 \\
\hline \multirow{3}{*}{ Particulars } & \multicolumn{9}{|c|}{ MUSCULARIS EXTERNA ( thickness in mm) } \\
\hline & \multicolumn{3}{|c|}{ DUODENUM } & \multicolumn{3}{|c|}{ JEJUNUM } & \multicolumn{3}{|l|}{ ILEUM } \\
\hline & 1 st tri & 2nd tri & 3rd tri & 1 st tri & 2nd tri & 3rd tri & 1 st tri & 2nd tri & 3rd tri \\
\hline MEAN & 538.8 & 248.7 & 463.2 & 255.9 & 228.9 & 273.6 & 353.8 & 183.8 & 207.2 \\
\hline MINIMUM & 334.8 & 195.0 & 334.8 & 107.7 & 158.4 & 180.9 & 270.5 & 112.9 & 149.0 \\
\hline MAXIMUM & 497.4 & 375.4 & 550.1 & 340.4 & 361.9 & 443.1 & 418.9 & 253.0 & 275.1 \\
\hline S D & 55.8 & 50.9 & 64.0 & 74.6 & 68.1 & 84.2 & 54.5 & 53.7 & 39.3 \\
\hline
\end{tabular}


Mucosa: Duodenal mucosa seemed to be thicker in the first trimester. During second trimester, the mucosa is seen to be decreased in its thickness. And in the third trimester, the mucosa is again observed to be thicker than in the first two trimesters. The thickness increased both because of development of epithelium, villi and increase in thickness of lamina propria.

The thickness of the mucosa in the jejunum does not show much difference between the first two trimesters. But in the third trimester mucosa seemed to be very thick. The thickness increased here because of development of epithelium, villi.

The mucosal layer of the ileum increases in its thickness during the first and third trimester while its thickness decreases during the second trimester. The thickness increased here because of development of epithelium, villi (Fig. 1).

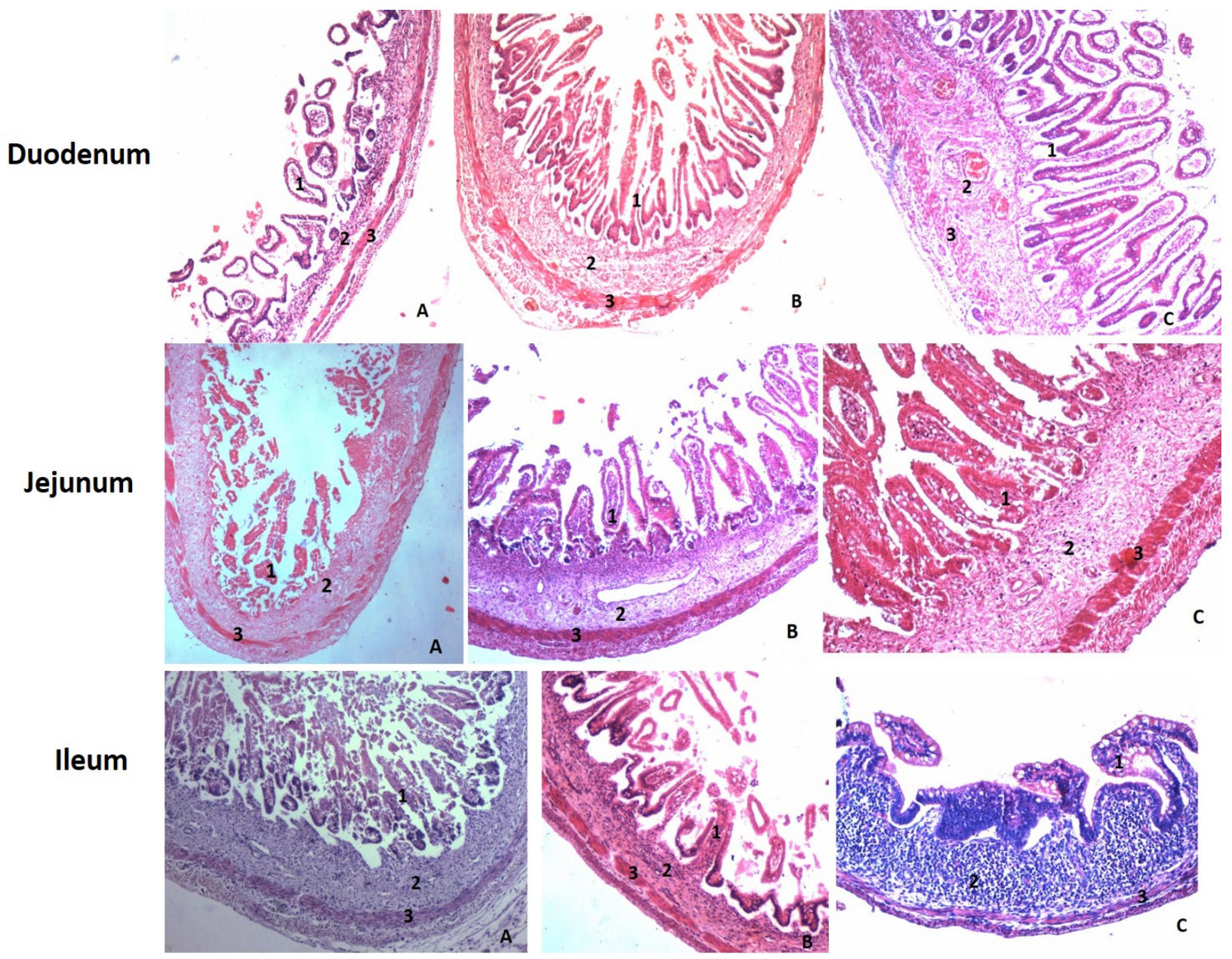

Figure 1. H\&E stained section $(10 \mathrm{x})$ of duodenum, jejunum and ileum during all three trimesters. (A. First trimester, B. $2^{\text {nd }}$ Trimester, C. $3^{\text {rd }}$ Trimester. 1- Mucosa, 2- Submucosa, 3 - Muscularis Externa).

The thickness of mucosal layer in the first trimester in in all three parts of the small intestines, duodenum, jejunum and ileum, seemed to be within the same range. In the second trimester, values of all the three are declined slightly. And in the third trimester, the value of the thickness of the jejunal mucosa is observed to be increased than the other two layers. This shows that there is no significant difference between duodenum and jejunum. Since, the ileum is the last part it is taking time to develop. However some significance is seen between jejunum and ileum as the p-value is less than 0.05. (Fig. 2)

Submucosa: The thickness of the submucosa in duodenum shows the same thickness pattern as the duodenal mucosa during three trimester. The thickness pattern of submucosa in the jejunum shows the same results as the jejunal mucosa. But, the submucosal layer in the ileum shows no much difference during all three trimesters. The thickness of submucosal layer during all the trimester is moreover the same. (Fig. 1) 
There is significant difference in the thickness of the submucosa in the first trimester between duodenum and ileum. However, in the second trimester, there is no significant difference between all the three parts of small intestine.

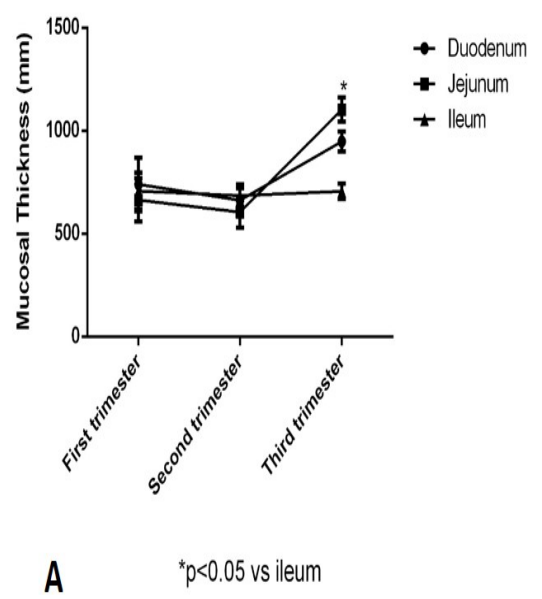

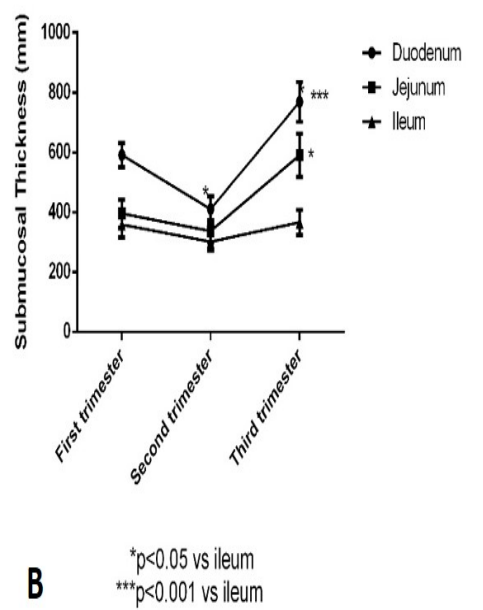

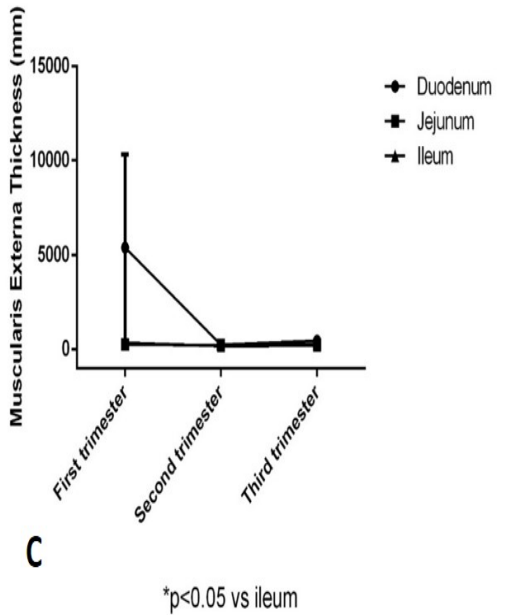

Figure 2. Showing the thickness \& significance of mucosa, submucosa and muscularis externa in small intestine during all three trimesters.

Muscularis externa: The muscularis externa of duodenum was relatively thicker in the first trimester than in the other two trimesters. The muscle layer in the jejunum is thinner than the muscle layer of the duodenum. And it shows constant thickness during all three trimesters. In the ileum, the thickness in the first trimester seemed to be increased than the thickness in the second and third trimester. (Fig. 1)

There is no significant change in the thickness of muscularis externa in the first trimester as the
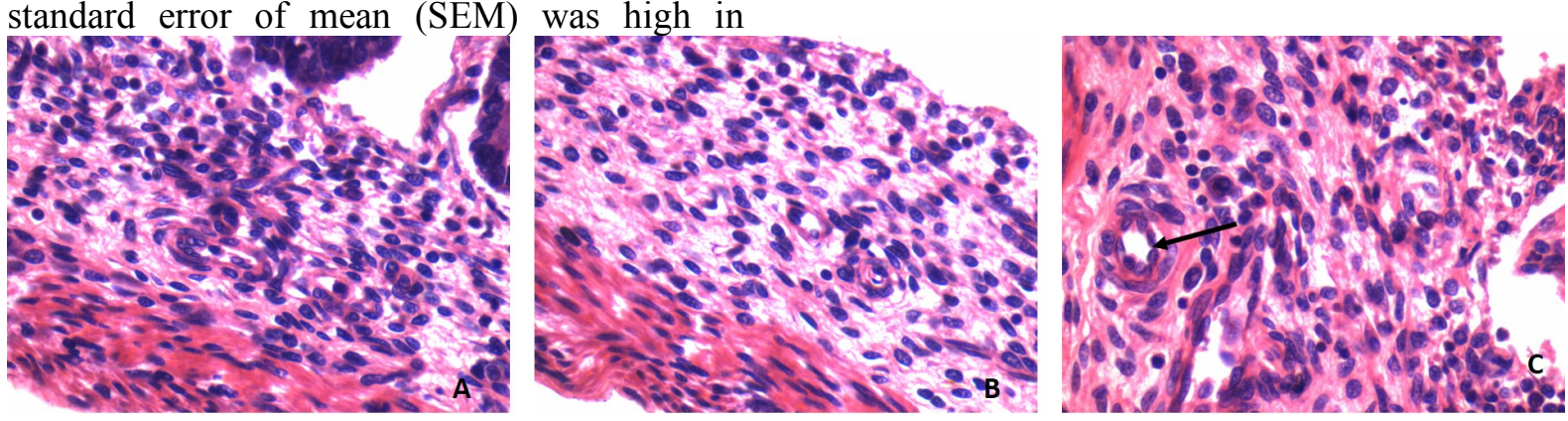

Figure 3. H\&E section (40x) showing the appearance of Brunner's gland in the duodenal submucosa in $3^{\text {rd }}$ Trimester pointed by an arrow. (A. First trimester, B. $2^{\text {nd }}$ Trimester, C. $3^{\text {rd }}$ Trimester).

Appearance of Peyers's patches: In the present study, we found discrete lymphoid tissues throughout the intestine in the submucosal layer. Peyer's patches are formed during the third trimester of foetal development. The lymphoid duodenum. In the second trimester, thickness of jejunum is significantly higher than the ileum with p-value less than 0.05 . In the third trimester, both duodenal and jejunal wall are significantly higher and thicker than those of the ileum. (Fig. 2)

Appearance of Brunners's gland: Brunner's glands are small and begin to develop in the submucosa of duodenum in 3rd trimester of the human fetus. There were no Brunner's glands seen in $1^{\text {st }}$ and $2^{\text {nd }}$ trimester (Fig. 3 ).

tissue was less observed in the 1st trimester and during the second trimester it gradually increased in its appearance and in the 3rd trimester it was highly increased and the patches began to form (Fig. 4). 

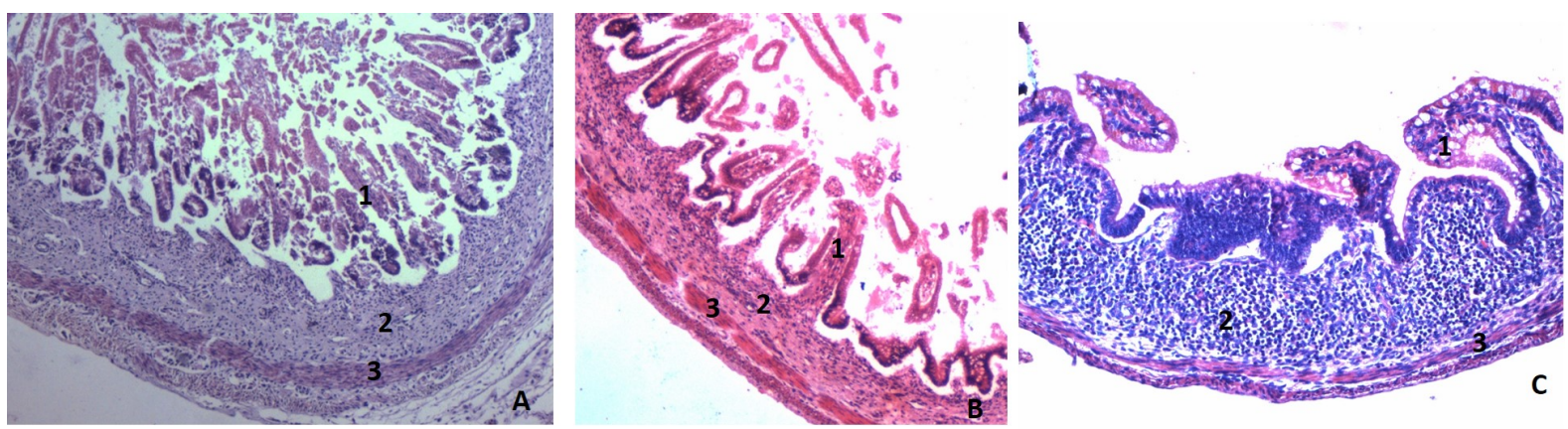

Figure 4. H\&E section (10x) showing the appearance of Peyer's patches in the submucosa of ileum in $3^{\text {rd }}$ Trimester. (A. First trimester, B. $2^{\text {nd }}$ Trimester, C. $3^{\text {rd }}$ Trimester. 1- Mucosa, 2- Submucosa, 3 - Muscularis Externa).

\section{DISCUSSION}

Sufficient nourishment which is a critical factor to the health and well-being of the newborn infant, depends on the digestive and absorptive capabilities of the intestinal epithelial cells at birth. These cells should also act as a "barrier" to the nonspecific intake and transport of luminal macromolecules. These digestives, absorptive, and barrier functions all depend on the structural maturity of the intestinal mucosa. This structural maturity in turn depend on numerous factors which include: (a) the types of cells present; (b) the distribution, composition, and organization of the intracellular organelles inside these various types of cells; and (c) the existence and continuity of an epithelial barrier with tight junctional complexes between nearby luminal cells. Information of this development progression will give understandings into the functional potential of the intestinal tract at various gestational ages. (6)

Drozdowski, et al (2010), in their study has mentioned that the development of small intestine comprises of three stages which is, a) morphogenesis and cell proliferation, 2) cell differentiation, and 3) functional maturation. And the epithelial lining of gastrointestinal tract in human begins in the $3^{\text {rd }}$ week of gestation. (3) In the present study we observed the development of the intestinal wall during the three trimesters of foetal development and we have observed that the first trimester is the stage where the morphogenesis, cell proliferation, and differentiation begins. However, the villi formation is still under development. The thickness of other layers of intestinal wall also keep increasing in size.

Bagyánszki $M$ studied the thicknesses of the epithelium, the submucosa, the muscular layers and the myenteric ganglia in resin-embedded semi-thin sections of human fetal intestine of 12 and 18 weeks. He noticed a gradual increase in the thickness of the intestinal wall between 12 and 18 weeks of gestation. (7) But in our study we observed that in the first trimester, all the three layers mucosa, submucosa and muscularis externa were comparatively thicker than the second trimester.

Schumacher et al, (2004) reported that in humans, the Brunner's glands begin to differentiate from the bases of the crypts of Lieberkühn just distal to the gastro-duodenal junction after about 3-5 months of intrauterine life (8). In the present study, we observed that the Brunner's glands are small and begin to develop in the submucosa in the 3rd trimester of the human fetus.

Botros $\mathrm{KG}$ et al found that at 12 weeks the initial development of Brunner's glands starts. At 16 weeks, they start originating from the ends of the most proximal crypts of the duodenum. From 20 weeks to full term, the Brunner's glands developed in a progressive fashion starting in the proximal part of the duodenum near the pyloroduodenal junction. (9) In the present study, we observed that the Brunner's glands are small and begin to develop in the submucosa in the 3rd trimester of the human fetus.

Cornes (1965), in their study observed that, ileum contained numerous Peyer's patches compared to jejunum which is obvious in nature of small intestine. Although, the jejunum also contain few lymphoid tissues, they mention that the duodenal patches were also observed in some of the specimens. They mention that, the more patches in the ileum were observed in the proximal part of it, which they measured as the longest patch was $9.9 \mathrm{~cm}$ in length. They also mentioned that the Peyer's patches are well developed in human small intestine in early fetal life. The fetal human small intestine contains in average 60 Peyer's patches before week 30 of gestation and their number steadily increase 
reaching a maximum of 240 at puberty (10). In the present study, we found that discrete lymphoid tissues were present throughout the intestine in the submucosal layer. In the third trimester of the fetus, Peyer's patches were formed. The lymphoid tissue was less observed in the $1^{\text {st }}$ trimester and gradually in the $2^{\text {nd }}$ trimester it started increasing and in the $3^{\text {rd }}$ trimester it was highly increased and the patches began to form.

Baginskys et al found distinct groups of $\mathrm{T}$ and B cells in the small intestine at 14-16 weeks of gestation. At week 19, these groups mature into identifiable Peyer's patches and they become visible macroscopically at week 24 , but there was no germinal centers. The Peyer's patches grow rapidly after birth, when the intestines are exposed to bacteria and antigens. (11) In our study the lymphoid tissue was less observed in the 1st trimester and gradually in the 2nd trimester it started increasing and in the 3rd trimester it was highly increased and the patches began to form.

Spencer $\mathrm{J}$ et al found that at 11 weeks gestation clusters of cells which express CD4 are present in fetal ileum but they are probably macrophages. They found that aggregates of lymphoid tissue are apparent from 14 weeks gestation which contain $\mathrm{T}$ cells of helper/inducer and suppressor/cytotoxic phenotype. Both B and $\mathrm{T}$ cells are present at 16 weeks but with no cellular zonation. By 19 weeks, distinct follicles of $\mathrm{B}$ cells are present surrounded by $\mathrm{T}$ cells of helper/inducer and suppressor/cytotoxic phenotype. (12) In our study the lymphoid tissue was less observed in the 1 st trimester and gradually in the 2 nd trimester it started increasing and in the 3rd trimester it was highly increased and the patches began to form.

Grand RJ et al told that brunner's gland develop in duodenum at 14 weeks and Peyer's patches in ileum at 20 weeks. (4) In the present study, we found that the lymphoid tissue was less observed in the 1st trimester and gradually in the 2nd trimester it started increasing and in the 3rd trimester it was highly increased and the patches began to form. We also found that the Brunner's glands are small and begin to develop in the submucosa in the 3 rd trimester of the human fetus.

In the present study we observed that in the first trimester, all the three layers mucosa, submucosa and muscularis externa were comparatively thicker than the second trimester, which could be because of the rotatory movement of the gut and the absorption mechanism. The second trimester seems to be the resting period as the intestine takes up its final position in the abdominal cavity by $13^{\text {th }}-14^{\text {th }}$ week of development. Again in the third trimester thickness of the layers in the intestinal wall were observed to be increased. This could be because of the increase cell turnover and the arrangement of the collagen fibers as to support the mucosa and the muscularis externa. We have also observed that the Brunner's glands are small and begin to develop in the submucosa of duodenum in 3rd trimester of the human fetus and the discrete lymphoid tissues were present throughout the intestine in the submucosal layer. Peyer's patches are formed in the third trimester of the fetus. The lymphoid tissue was less observed in the 1st trimester and gradually in the 2nd trimester it started increasing and in the 3rd trimester it was highly increased and the patches began to form.

The morphology and histological structure of the small intestine are examined in fetuses to assess the peculiarities of intestinal differentiation before birth. The study of histological differentiation helps us to understand the morphological changes during the development of human fetal small intestine. This will help the gastroenterologist to identify any pathological and physiological changes in the small intestine which can happen later in life.

\section{REFERENCES}

1. Singh I. Histology: textbook of human histology with color atlas and practical guide, 7th Edition. Revised and edited by Neelam Vasudeva, Sabita Mishra, 2014.

2. Kulkarni NV. Clinical Anatomy-A Problem Solving Approach, $3^{\text {rd }}$ Edition, 2016.

3. Drozdowski LA, Clandinin T, Thomson A BR. World J gastroenterol. 2010; 16(7):787-799.

4. Grand RJ, Watkins JB, and Tort FM. Development of the human gastrointestinal tractA Review. Gastroenterology. 1976, 70:790-810.

5. Carleton HM, Drury RAB, Wallington EA. Carleton's Histological technique. $5^{\text {th }}$ Edition, Oxford University Press, 1980.

6. Colony PC. Successive Phases of Human Fetal Intestinal Development. Nutritional Adaptation of the Gastrointestinal Tract of the Newborn, edited by N. Kretchmer and A. Minkowski. Nestle, Vevey/Raven Press, New York, 1983; 3.

7. Bagyánszki M, Kovács EG, Resch BA, Román V, Resch BE and Fekete E. Computer-aided morphometric analysis of the developing 
concentric structure of the human fetal intestinal tube. Histol Histopathol. 2002; 17: 731-737.

8. Schumacher U, Duku M, Katoh M, Jörns J, Krause WJ. Histochemical similarities of mucins produced by Brunner's gland and pyloric glands: A comparative study. The anatomical record. 2004; 278A:540-550.

9. Botros KG, el-Hady SL, el-Mohandes EA. Prenatal development of the human Brunner's glands. Anat Anz. 1990; 171(1):23-30.
10. J.S.Cornes. Number, size, and distribution of Peyer's patches in the human small intestine. Gut. 1965; 6: 225.

11. Baginsky A. Untersuchungen uber den Darmkanal des menslichen Kindes. Virchows Arch. path. Anat.1882; 89: 64-94.

12. Spencer J, MacDonald TT, Finn T, Isaacson PG. The development of gut associated lymphoid tissue in the terminal ileum of fetal human intestine. Clin Exp Immunol. 1986; 64(3):536-43. 